\title{
Targeting anti-apoptotic BCL-2 family proteins for cancer treatment
}

\author{
Xuan Zhang1 ${ }^{1}$, Xingui Liu², Daohong Zhou² \& Guangrong Zheng*,1 (iD \\ ${ }^{1}$ Department of Medicinal Chemistry, College of Pharmacy, University of Florida, 1333 Center Drive, Gainesville, FL 32610, USA \\ ${ }^{2}$ Department of Pharmacodynamics, College of Pharmacy, University of Florida, 1333 Center Drive, Gainesville, FL 32610, USA \\ *Author for correspondence: zhengg@cop.ufl.edu
}

\begin{abstract}
“A number of strategies, such as BCL- $X_{L}$-targeting ADCs and BCL- $X_{L}$ PROATCs, have been developed to circumvent the on-target thrombocytopenia associated with $B C L-X_{L}$ inhibition. Similar strategies could also be employed to reduce the potential on-target cardiac toxicity associated with MCL-1 inhibition."
\end{abstract}

First draft submitted: 2 January 2020; Accepted for publication: 13 January 2020; Published online: 21 February 2020

Keywords: apoptosis $\bullet \mathrm{BCL}-2 \bullet \mathrm{BCL}-\mathrm{X}_{\mathrm{L}} \bullet$ cancer treatment $\bullet \mathrm{MCL}-1$

Apoptosis, also known as programmed cell death, contributes to cellular homeostasis, normal development and clearance of abnormal cells [1]. Dysregulation of apoptotic pathways plays an important role in tumorigenesis and is considered to be one of the hallmarks of cancer [2]. Moreover, resistance to apoptosis is associated with desensitization to conventional cytotoxic and targeted therapies. Therefore, the induction of cell death by targeting apoptotic pathways is an attractive therapeutic strategy. BCL-2 family proteins are the key regulators of the mitochondria-mediated apoptotic pathway and can be divided into three subsets; pro-apoptotic BH3-only proteins (apoptosis initiators, such as BIM, BID and PUMA), pro-apoptotic proteins BAK and BAX (apoptosis effectors) and anti-apoptotic proteins (apoptosis gatekeepers, such as BCL-2, BCL-X $\mathrm{L}_{\mathrm{L}}, \mathrm{MCL}-1$ and BCL-w) [3]. Among those, several anti-apoptotic members including BCL-2, BCL- $\mathrm{X}_{\mathrm{L}}$, and MCL-1 are well-validated anticancer targets. Displacement of pro-apoptotic proteins from the binding groove of anti-apoptotic members results in homooligomerization of BAK and BAX, permeabilization of mitochondrial outer membrane, release of cytochrome $c$ and activation of caspases to trigger apoptosis [1].

\section{The development of first FDA approved BCL-2 antagonist}

Targeting protein-protein interactions with small molecules has long been considered as a very challenging task for medicinal chemists. However, with fragment-based drug design approach coupled with novel drug discovery techniques such as nuclear magnetic resonance (NMR) spectroscopy and x-ray crystallography, many successful examples have been reported. The discovery of ABT-737 [4], the first potent dual inhibitor of BCL-2 and BCL-X was based on the above-mentioned approaches. Due to its unfavorable pharmacokinetic properties, researchers at Abbvie developed the orally bioavailable successor, navitoclax (ABT-263), which entered clinical trials in 2006. However, navitoclax induces rapid and dose-dependent thrombocytopenia [3]. This clinical observation is in accordance with preclinical evidence that platelets depend on BCL- $\mathrm{X}_{\mathrm{L}}$ for survival [5].

To overcome the on-target and dose-limiting toxicity associated with BCL- $\mathrm{X}_{\mathrm{L}}$ inhibition, a highly selective BCL2 inhibitor, venetoclax (ABT-199), was developed by Abbvie. Venetoclax shows robust anti-tumor effects against various hematological malignancies while sparing platelets. It was approved by the US FDA for the treatment of chronic lymphocytic leukemia and small lymphocytic lymphoma as a single agent and for acute myeloid leukemia in combination with azacitidine, decitabine, or low-dose cytarabine [6].

\section{Ongoing strategies in targeting $\mathrm{BCL}-\mathrm{X}_{\mathrm{L}}$}

Venetoclax has remarkable clinical performance and great potential for further investigation, both as monotherapy and in combination with other drugs to treat hematological malignancies. However, it has limited applications for 
the treatment of solid tumors. In contrast, $\mathrm{BCL}-\mathrm{X}_{\mathrm{L}}$ is overexpressed in various solid tumors and a subset of leukemia and lymphoma cancerous cells. Bioinformatics analyses also reveal a strong correlation between the levels of BCL- $\mathrm{X}_{\mathrm{L}}$ expression and resistance to chemotherapies [7]. Therefore, even though the clinical applications of ABT-263 as a single agent is hampered by its dose-limiting platelet toxicity, several clinical trials to circumvent platelet toxicity of ABT-263 through combination with chemotherapeutic agents are ongoing [8]. In addition, APG-1252, a prodrug derived from a $\mathrm{BCL}-2 / \mathrm{X}_{\mathrm{L}}$ dual inhibitor, is designed to minimize the drug exposure to platelets and is currently in clinical trials to treat patients with small cell lung cancer and other solid tumors [9]. Recently, utilizing a novel drug delivery technology, AstraZeneca’s DEP ${ }^{\circledR}$ BCL-2/X $\mathrm{X}_{\mathrm{L}}$ conjugate, AZD-0466, received FDA authorization for clinical trial [10].

It is worth noting that the exacerbation of neutropenia was observed when ABT-263 was combined with docetaxel clinically, which is likely attributed to the BCL-2 inhibitory effect of navitoclax [11]. By contrast, selective BCL-X inhibitor A-1331852 enhanced the efficacy of docetaxel while sparing neutropenia, indicating the potential and benefit to recruit selective BCL- $\mathrm{X}_{\mathrm{L}}$ inhibitor for further investigation. By converting a selective BCL- $\mathrm{X}_{\mathrm{L}}$ inhibitor into an antibody-drug conjugate (ADC), the selectivity to specific cancer cells over platelets can be improved. A representative ADC, ABBV-155, which targets B7H3-expressing cancer cells, is currently in Phase I clinical trial for the treatment of advanced solid tumors [12]. However, no small-molecule BCL- $\mathrm{X}_{\mathrm{L}}$ selective antagonists have been advanced for clinical evaluation.

A novel strategy to reduce on-target toxicity is to utilize proteolysis targeting chimera (PROTAC) technology. The rationale is based on the differentially expressed E3 ligases in different cells/tissues. Thus, cell/tissue specificity could be achieved by converting an inhibitor to a PROTAC. In proof of concept studies, both Von HippelLindau (VHL) cullin-2 and cereblon (CRBN) cullin-4A RING E3 ligases, which were found to be minimally expressed in human platelets, have been recruited to degrade BCL- $\mathrm{X}_{\mathrm{L}}[13,14]$. The preliminary data support the reduction of on-target platelet toxicity for both CRBN-based and VHL-based PROTAC degraders. Further, the lead candidate, DT2216, a VHL-based PROTAC derived from ABT-263, achieved better potency to a variety of BCL- $\mathrm{X}_{\mathrm{L}}$ dependent cancer cells with a significantly improved therapeutic window than its parent compound ABT-263 [14].

\section{MCL-1 inhibition for cancer therapy}

Targeting MCL-1 for cancer treatment has also been explored for over a decade. Several hematological cancers, including multiple myeloma and acute myeloid leukemia and some solid tumors (e.g., hepatocellular carcinoma and non-small-cell lung cancer) rely on MCL-1 for survival [15], while showing modest response to BCL-2 inhibition. Earlier reported MCL-1 inhibitors in the literature are often with low selectivity over BCL-2 and/or BCL-X $\mathrm{X}_{\mathrm{L}}$ or work via alternative mechanisms rather than direct MCL-1 inhibition.

Inspired by the increasing biological evidence that a variety of cancer cells depend on MCL-1 for their viability, several highly selective and potent MCL-1 inhibitors have been developed recently [16]. In comparison with BCL-X and BCL-2 inhibitors, MCL-1 antagonists are smaller in size but more complicated in structure. To occupy the large and shallow binding groove as much as possible to achieve sufficient on-target cellular efficacy, numerous chiral centers, atropisomerism and macrocyclization have been introduced into the scaffold to maintain a favorable binding conformation. Tremendous efforts in lead optimization yielded several highly selective MCL-1 inhibitors with binding affinity at picomolar levels, which is required for on-target cellular activity [15].

It is important to realize that the preclinical evaluation of MCL-1 inhibitors is challenging since a variety of MCL-1 inhibitors have less binding affinities to mice MCL-1 than to human MCL-1. As a result, the in vivo studies of MCL-1 inhibitors using wide-type mice may not be suitable for the prediction of the drug efficacy and toxicity. The introduction of human MCL-1 knock-in mice mostly resolved this issue, and so far, five MCL-1 inhibitors (AMG-176, AMG-397, AZD5991, S64315/MIK665 and ABBV-467) have entered Phase I clinical trials [17].

Late in 2019, the FDA handed out a hold for AMG-397 in clinical trials due to 'safety signal for cardiac toxicity'. Meanwhile, Amgen has voluntarily halted enrollment for another early-stage test for AMG-176 [18]. Given that prosurvival role of MCL-1 may be essential for normal cardiac myocyte mitochondrial activity [17], the observations of clinical cardiac events are likely because of the on-target toxicity. If that is the case, conventional MCL-1 inhibitors may all have limited therapeutic window regardless of their chemotypes. However, the fate of the MCL-1 inhibitors will likely be determined by the outcomes of the currently ongoing clinical trials. 


\section{Future perspective}

The FDA approval of BCL-2 selective inhibitor, venetoclax, is a milestone in targeting the intrinsic apoptotic pathway for cancer treatment. Further clinical trials of venetoclax hold the potential of additional applications in hematologic cancers. Small molecules that target BCL- $\mathrm{X}_{\mathrm{L}}$ or MCL-1 have the potential of broader applications in cancer treatments compared with BCL-2 inhibitors. However, the clinical development of BCL-X $\mathrm{X}_{\mathrm{L}}$ or MCL-1 inhibitors has been hampered by their on-target, dose-limiting toxicities. A number of strategies, such as BCL$\mathrm{X}_{\mathrm{L}}$-targeting ADCs and BCL- $\mathrm{X}_{\mathrm{L}}$ PROATCs, have been developed to circumvent the on-target thrombocytopenia associated with BCL- $\mathrm{X}_{\mathrm{L}}$ inhibition. Similar strategies could also be employed to reduce the potential on-target cardiac toxicity associated with MCL-1 inhibition. MCL-1 PROTACs have been recently developed $[19,20]$. It will be interesting to evaluate whether these degraders have the benefit of reduced on-target toxicity.

\section{Financial \& competing interests disclosure}

This work was supported in part by NIH grants R01CA211963, R01CA219836, R01CA242003 and R21 CA223371. The authors have no other relevant affiliations or financial involvement with any organization or entity with a financial interest in or financial conflict with the subject matter or materials discussed in the manuscript apart from those disclosed.

No writing assistance was utilized in the production of this manuscript.

\section{References}

1. Singh R, Letai A, Sarosiek K. Regulation of apoptosis in health and disease: the balancing act of BCL-2 family proteins. Nat. Rev. Mol. Cell Biol. 20(3), 175-193 (2019).

2. Hanahan D, Weinberg RA. Hallmarks of cancer: the next generation. Cell 144(5), 646-674 (2011).

3. Lessene G, Czabotar PE, Colman PM. BCL-2 family antagonists for cancer therapy. Nat. Rev. Drug Discov. 7(12), 989-1000(2008).

4. Oltersdorf T, Elmore SW, Shoemaker AR et al. An inhibitor of BCL-2 family proteins induces regression of solid tumors. Nature 435(7042), 677-681 (2005).

5. Zhang H, Nimmer P, Tahir S et al. BCL-2 family proteins are essential for platelet survival. Cell Death Differ. 14(5), 943-951 (2007).

6. Souers AJ, Leverson JD, Boghaert ER et al. ABT-199, a potent and selective BCL-2 inhibitor, achieves antitumor activity while sparing platelets. Nat. Med. 19(2), 202-208 (2013).

7. Amundson SA, Myers TG, Scudiero D, Kitada S, Reed JC, Fornace AJ. An informatics approach identifying markers of chemosensitivity in human cancer cell lines. Cancer Res. 60(21), 6101-6110 (2000).

8. Anantram A, Degani M. Targeting cancer's Achilles' heel: role of BCL-2 inhibitors in cellular senescence and apoptosis. Future Med. Chem. 11(17), 2287-2312 (2019).

9. Wu Y, Lakhani N, Boyer M et al. A Phase I study of novel BCL-2/BCL-xL inhibitor APG-1252 in patients with advanced SCLC or other solid tumor. J. Thorac. Oncol. 13(12), S1048-S1049 (2018).

10. Starpharma. FDA authorisation of first-in-human clinical trial with AstraZeneca's DEP ${ }^{\circledR}$ product AZD0466 (2019). www.starpharma.com/news/story/fda-authorisation-of-first-in-human-clinical-trial-with-astrazenecaas-dep-product-azd0466

11. Leverson JD, Phillips DC, Mitten MJ et al. Exploiting selective BCL-2 family inhibitors to dissect cell survival dependencies and define improved strategies for cancer therapy. Sci. Transl. Med. 7(279), 279ra240 (2015).

12. FDA. A study with ABBV-155 alone and in combination with taxane therapy in adults with relapsed and/or refractory solid tumors (2018). www.clinicaltrials.gov/ct2/show/NCT03595059

13. Zhang X, Thummuri D, He Y et al. Utilizing PROTAC technology to address the on-target platelet toxicity associated with inhibition of BCL-X L. Chem. Commun. 55(98), 14765-14768 (2019).

14. Khan S, Zhang X, Lv D et al. A selective BCL-X $\mathrm{L}$ PROTAC degrader achieves safe and potent antitumor activity. Nat. Med. 25(12), 1938-1947 (2019).

15. Fletcher S. MCL-1 inhibitors - where are we now (2019)? Expert Opin. Ther. Pat. 29(11), 909-919 (2019).

16. Xiang W, Yang C-Y, Bai L. MCL-1 inhibition in cancer treatment. Onco Targets Ther. 11, 7301-7314 (2018).

17. Hird AW, Tron AE. Recent advances in the development of MCL-1 inhibitors for cancer therapy. Pharmacol. Ther. 198, 59-67 (2019).

18. ASHClinicalNews. FDA places trials of MCL-1 inhibitor on clinical hold (2019). www.ashclinicalnews.org/news/latest-and-greatest/fda-places-trials-mcl-1-inhibitor-clinical-hold/

19. Wang Z, He N, Guo Z et al. Proteolysis targeting chimeras for the selective degradation of MCL-1/BCL-2 derived from nonselective target binding ligands. J. Med. Chem. 62(17), 8152-8163 (2019).

20. Papatzimas JW, Gorobets E, Maity R et al. From inhibition to degradation: targeting the anti-apoptotic protein myeloid cell leukemia 1 (MCL1). J. Med. Chem. 62(11), 5522-5540 (2019). 
